\title{
Peripheral regulation of stress and fear responses in pigs from tail-biting pens
}

\author{
Manja Zupan ${ }^{1 *}$, Adroaldo José Zanella²
}

\begin{abstract}
${ }^{1}$ University of Ljubljana, Biotechnical Faculty, Department of Animal Science, Domžale, Slovenia.
${ }^{2}$ Universidade de São Paulo, Faculdade de Medicina Veterinária e Zootecnia, Departamento de Medicina Veterinária Preventiva e Saúde Animal, Pirassununga, SP, Brazil.
\end{abstract}

\begin{abstract}
This study focused on stress physiology by characterizing cortisol responses to stressors in tail biters $(n=10)$, victims $(n=10)$, and control pigs $(n=10)$ of two different breeds (Landrace $\times$ Yorkshire, LY; Landrace/Yorkshire $\times$ Landrace/ Duroc; LYLD) and sexes (females and castrated males). We exposed pigs to 10 min of isolation with a human at the farm and transported them to a controlled environment. There, the behaviour was registered for 10 min during sessions when subjected to a novel object test and to a novel arena test. Sampling times of salivary cortisol were in all the fear tests before testing and 60 min thereafter, while for transportation as well $120 \mathrm{~min}$ after the beginning of transportation. We additionally measured cortisol at 7:00 and 16:00 h during three days following transportation. The basal stress axis activity followed a distinct diurnal rhythm between sexes and breeds, with castrated males having higher cortisol level than females and LY pigs higher than LYLD pigs. Following isolation at the farm and transportation, the concentration of salivary cortisol was higher in LY than in LYLD pigs. Pigs considered the exposure to a novel arena, but not to a novel object, stressful by showing a cortisol level after testing higher than before testing. The results suggest a genotypic effect on sensitivity to stress in pigs that have performed tail biting, have been victimized, or have not been involved in tail biting.
\end{abstract}

Key Words: damaging behaviour, emotions, genotype, salivary cortisol, stressors

\section{Introduction}

Tail biting in pigs is a behavioural disorder characterized by oral manipulation of the tail of another pig, often resulting in lesions (Taylor et al., 2010). Pigs involved in tail biting suffer from inflammatory responses (Heinonen et al., 2010) and fearfulness (Zupan et al., 2012, 2016), thus having impaired physical and psychological health.

It is a general consensus that the autonomic and the endocrine activities mediate the peripheral components of psychological states such as stress or fear and anxiety. Fearfulness, the predisposition to show fear- and anxietyrelated responses to a range of potential and real threats (Forkman et al., 2007), has implications for the ability to cope with specific types of environmental changes and

Received: July 29, 2016

Accepted: October 18, 2016

*Corresponding author: manja.zupan@bf.uni-lj.si

http://dx.doi.org/10.1590/S1806-92902017000100006

How to cite: Zupan, M. and Zanella, A. J. 2017. Peripheral regulation of stress and fear responses in pigs from tail-biting pens. Revista Brasileira de Zootecnia 46(1):33-38

Copyright (C) 2017 Sociedade Brasileira de Zootecnia. This is an Open Access article distributed under the terms of the Creative Commons Attribution License (http://creativecommons.org/licenses/by/4.0/), which permits unrestricted use, distribution, and reproduction in any medium, provided the original work is properly cited. challenges. Fearful pigs may thus have a predisposition to become more stressed by environmental changes. There is some evidence that individual differences in ability to adjust to environmental challenges may lead to sub optimal development of stress-axis activity after restraint stress or exposure to novelty (Herman et al., 2005; Fries et al., 2005).

For this reason, we tested pigs for the effects of biting tails and having tails bitten on measures of fear-related behavioural and physiological responses. We documented that the activity of the autonomic nervous system, especially the suppression of parasympathetic tone, indicates that some pigs may have a dysfunctional autonomic regulation (Zupan et al., 2012). Additionally, the results indicated that in tail-biting pens, pigs of different sexes and breeds possess different autonomic flexibility (Zupan et al., 2016). In this paper, we tested pigs in a human-response test, a novel object test, a novel arena test, and during transportation and assessed the activation of stress-axis, using a non-invasive method to measure salivary cortisol concentrations. By performing different behaviour responses to environmental situations (Zupan et al., 2012, 2016), we expected animals to express different stress susceptibility, with tail biters and victims to have higher cortisol levels than control pigs. Equally, by investigating diurnal rhythm in cortisol release, we expected the genotypic characteristics of the pigs to affect their circadian rhythm. 


\section{Material and Methods}

We studied the same pigs and commercial farms as described previously (Zupan et al., 2012, 2016). Farm visits followed a report of tail-biting outbreaks on three commercial Norwegian farms. Briefly, 30 pigs with intact tails were selected based on the frequency of tail biting during an observation period of $60 \mathrm{~min}$ on a farm. The experimenters selected up to two trios of one tail biter, a victim, and a control pig from the same pen. Tail biters $(\mathrm{n}=10)$ were observed to repeatedly bite the tails of other pigs. Victims $(n=10)$ were chosen based on tail damage and not bites received. Controls $(n=10)$ were neither observed biting tails nor had damaged tails. The pens were of mixed sex. There were females and castrated males. Pigs were of two crossbreeds, Landrace $\times$ Yorkshire (LY) and Landrace/Yorkshire $\times$ Landrace/Duroc (LYLD). A particular phenotype consisted of three castrated males (LY, $\mathrm{n}=2 ; \mathrm{LYLD}, \mathrm{n}=1)$ and seven females (LY, $\mathrm{n}=3$; LYLD, $\mathrm{n}=4$ ). The body weights (mean \pm standard error $\mathrm{kg}$ ) of tail

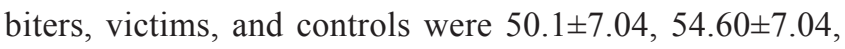
and 53.20 \pm 7.04 , respectively, with no significant differences (ANOVA: $\mathrm{F}_{2,30}=0.08 ; \mathrm{P}>0.10$ ).

Following selection at the farm, pigs were transported to the research facility using a trailer designed for transporting horses. The duration of transport ranged between 38 and $177 \mathrm{~min}$. The average daily temperature during the days of transportation was between -3.5 and $13.1{ }^{\circ} \mathrm{C}$. On arrival at the facility, pigs were weighed and housed individually in pens. They were tested in five blocks of six pigs per block, with each block consisting of two trios. Pens, $1.25 \times 1.79 \mathrm{~m}$ each, were arranged in two rows of three pens. Visual, olfactory, auditory, and limited tactile contact was allowed between adjacent pigs. Biters were in contact with biters, victims with victims, and controls with controls. Commercial pig diet and water were available ad libitum via a trough and water nipple, respectively. The temperature in the home pens ranged from 19 to $21^{\circ} \mathrm{C}$. In addition to natural light, artificial lights were on from 08:00 to 15:00 h.

To reveal potential differences in physiological responses, a battery of tests were applied to characterize the three pre-determined phenotypes. The day after the formation of trios, pigs were tested on a farm in a human test. They were tested for their behaviour to isolation in an adjacent pen, i.e. test arena, together with an unfamiliar stationary person wearing a white coat and green boots. Shortly before testing, a human entered the home pen of the selected pigs and took basal saliva sample as described below. Afterwards, a pig was gently taken into the test arena. After testing, the pig was led back to its home pen. Following completion of testing at the farm, pigs were tested at the research facility where their responses to a novel object in a novel-object test in the home pen and an isolation test in a novel-arena test were measured. Saliva samples were collected at several time points during the experiment. Firstly, they were collected during the human test on a farm and during the novel-object and the novelarena tests at the facility. Sampling times were similar in all the three tests: immediately before testing (basal measure) and $60 \mathrm{~min}$ thereafter (post-test measure). The time of the post-test measure was chosen on the basis of data showing an increase in cortisol response an hour after conducting intravenous injection of ACTH (adrenocorticotropic hormone) (Sautron et al., 2015). Secondly, saliva samples were taken before the transportation, 60 and $120 \mathrm{~min}$ after the beginning of transportation, from a farm to the research facility. Finally, three days following transportation, diurnal saliva samples were taken at 07:00 and 16:00 h.

The samples were obtained utilizing the cotton dental buds suspended on dental floss. Each pig was allowed to chew on the cotton buds for 20-30 s until buds were thoroughly moistened. The buds were placed into $15-\mathrm{mL}$ polypropylene centrifuge tubes and placed on ice. Within $1 \mathrm{~h}$ of collection, the saliva samples were centrifuged for $5 \mathrm{~min}$ at $1,000 \times g$ to extract the saliva. The saliva was pipetted to $1-\mathrm{mL}$ tubes and stored in a $-20^{\circ} \mathrm{C}$ freezer until assayed for cortisol. Samples were later thawed and assayed using an enzyme immunoassay kit (Assay Designs, Inc., U.S.A). Values of salivary cortisol concentration were reported in picogram per millilitre. For the saliva quality controls, the intra - and inter-assay coefficients of variation were 3.07\% and $3.13 \%$, respectively.

The statistical analysis was performed with the SAS package (Statistical Analysis System, version 9.1) using 30 animals, 10 per phenotype $(\mathrm{n}=30$, transportation, diurnal saliva), or less ( $n=21$, human test; $n=24$, novel-object test; $\mathrm{n}=27$, novel-arena test). Some pigs were removed from testing or excluded from the analysis due to either recording failure $(n=4)$, leg damage $(n=1)$, excessive reactivity to experimental protocol $(\mathrm{n}=1)$, or due to technical problems $(\mathrm{n}=3)$.

Results are shown as means and standard errors and all reported P-values are two-tailed. The data residuals were tested for normality (UNIVARIATE procedure) and when a normal distribution of the data could not be assumed, the data were log-transformed to achieve approximate normality. The data set from salivary cortisol concentrations were analyzed with analysis of variance by using the GLM procedure. Residuals followed normal distribution 
without (novel-object test, novel-arena test) or with ( $\log 10$; transportation, human test, diurnal saliva) transformation of data. The fixed effects of breed, sex, time (i.e. for transportation, the effect had three levels and for diurnal saliva and human test, it had two levels), and phenotype were tested for differences. We also considered the farm as additional fixed effect in the initial stage of the developing models, but by finding a strong non-significant effect on the cortisol responses, we excluded it from the following models. For the diurnal saliva concentrations, the effect of day was added. The effect of body weight was tested in each model as a covariate. When a significant effect was found, the LSMEANS and ESTIMATE statements were used to estimate the contrasts between factor levels and to compare their means. When more than two means needed to be compared, a multiple post-hoc Tukey-Kramer test was utilized to find the significant differences. Differences between the levels of the fixed effects were considered significant when $\mathrm{P} \leq 0.05$ or in tendency when $\mathrm{P}<0.10$.

To investigate Pearson correlation coefficients, procedure CORR was used to assess the relationship between the cortisol measures in all tested conditions between pigs of different breed, sex, or phenotype. In the text, only Bonferroni-corrected statistical significant values were reported $(\mathrm{P}<0.05, \mathrm{~B}: \mathrm{P}<0.01)$.

\section{Results}

In the human test and for the stressor transportation, the results revealed the effect of breed and time. When subjected to a human presence, LY pigs showed a tendency with higher cortisol concentration in comparison with LYLD pigs (Table 1). We also found a tendency with higher cortisol concentration before the animal was isolated, i.e. when in pen, than when isolated, i.e. alone in a pen. When subjected to transportation, pigs had the lowest concentrations before they were transported and the highest $60 \mathrm{~min}$ after the beginning of the transportation. Afterwards, the concentration started to return towards the basal concentrations. With the significant effect of breed, we found higher cortisol responses in LY pigs than in LYLD pigs. In the novel-object test, cortisol concentration was not affected by the investigated factors, while in the novel-arena test, the increase in cortisol concentration was significant after testing. Circadian morning values were as expected, higher than the afternoon values, but they were not affected by day. Pigs of different sexes and breeds differed in the circadian concentrations with castrated males having higher concentration when compared with females and LY higher levels when compared with LYLD.
There was a trend found with body weight affecting the circadian cortisol concentration $(\mathrm{P}<0.7 ; \mathrm{F}$-value $=3.62)$ and the concentration in the novel-arena test $(\mathrm{P}<10 ; \mathrm{F}$-value $=$ 2.88). Contrary to our expectations, no effect of tail-biting phenotype was detected on the cortisol release during different stressors.

Regarding the correlation analysis, it was revealed that in pigs from tail-biting pens the cortisol concentrations in the novel-arena and the novel-object tests were positively correlated with circadian concentrations ( $\mathrm{rs}>0.5$ and $\mathrm{rs}>0.4$, respectively). Positive correlation was also found between the concentrations in the novel-arena and the novel-object tests $(\mathrm{rs}>0.5)$. All these three positive correlations were found in LY pigs (all rs>0.5), but not in LYLD pigs. In victims, a strong positive correlation was found between the concentrations in the novel-arena test and the circadian concentrations $(\mathrm{rs}>0.7)$ and in controls, between the concentrations in the novel-object test and the circadian concentrations ( $r s>0.9)$. In pigs of different sexes, no significant correlations were found.

\section{Discussion}

This study highlights peripheral physiological responses of hypothalamic-pituitary-adrenal (HPA)-axis activation in pigs from tail-biting pens. One of our main results is finding no difference in cortisol level in phenotypes, which may indicate that all three phenotypes had similar adaptation mechanism to cope with different stressors. Phenotypes even had comparable diurnal cortisol patterns. However, interestingly, they had different basal heart rate variability (Zupan et al., 2012). Finding no cortisol difference during transportation in our study contradicts the findings of Valros et al. (2013), who reported that tail-bitten pigs show a lower cortisol response to the transport-induced stress than control pigs. In that study, pigs were selected from a single farm and control pigs were selected from the pens with no tail-biting outbreaks, which could explain the inconsistency between the studies. When assessing at the novel-object and the novel-arena test situations, it was interesting to see that, although the behaviour responses were indicative of emotional experiences and the cardiovascular responses were induced (Zupan et al., 2012, 2016), no difference in the activation of HPA-axis was found. We found similar results in pigs of different breeds and sexes. Despite the fact that our results are based on a small sample size, our data in the novel-object and the novel-arena tests were unexpected. To the knowledge of the authors, there is no research comparable to ours, done with the physiological responses of pigs from tail-biting pens 
Table 1 - Impact of factors on the salivary cortisol concentrations in pigs exposed to a battery of stressors

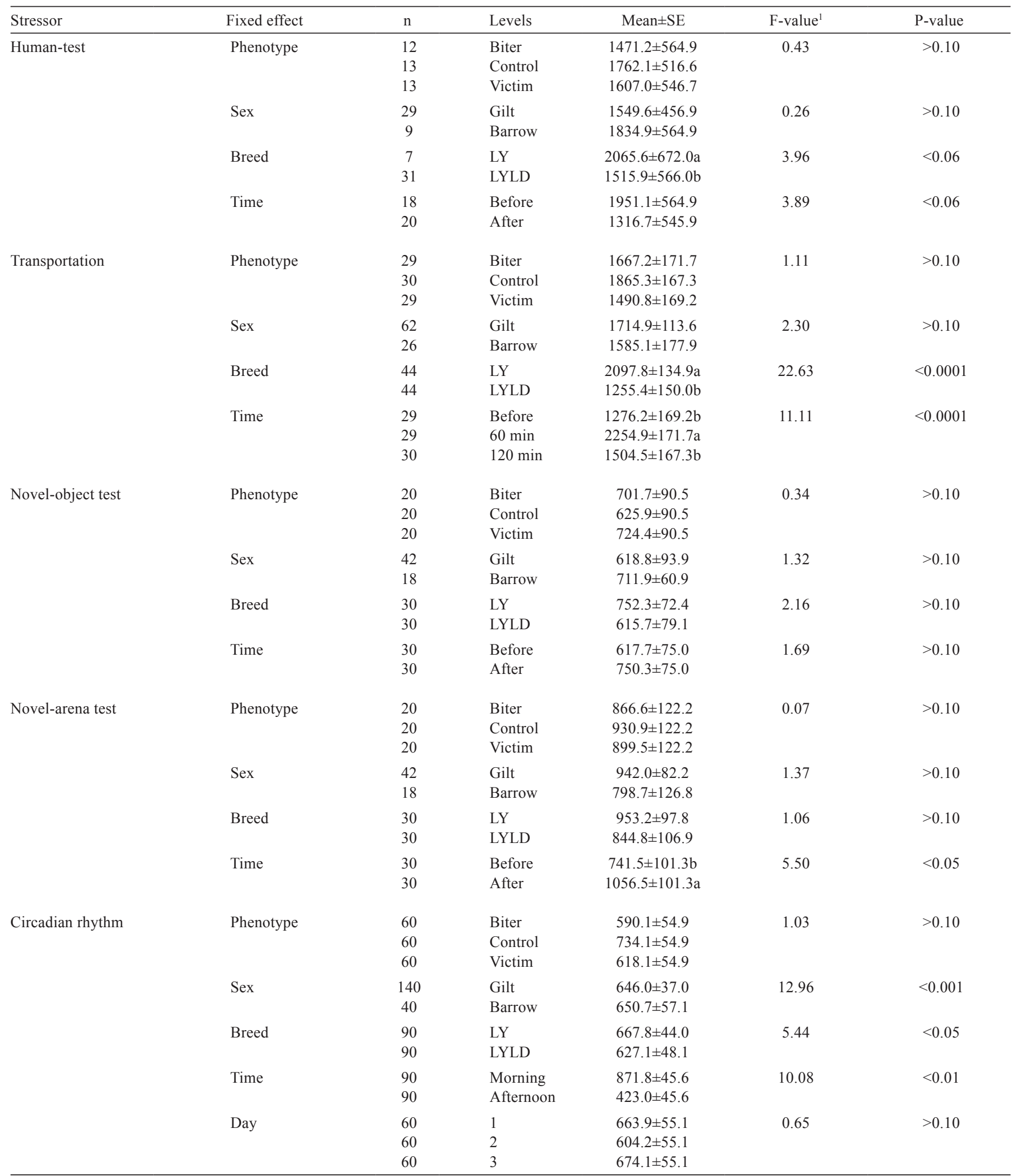

LY - Landrace $\times$ Yorkshire; LYLD - Landrace/Yorkshire $\times$ Landrace/Duroc; $n$ - number of recorded data; SE - standard error. a,b,c - Means in the same column with a different letter differ significantly (ANOVA, $\mathrm{P}<0.001$ )

${ }^{1}$ Analysis data were obtained by ANOVA. 
and of different phenotypes. In the latest study, focusing on behavioural and salivary cortisol responses to novelty in pigs from tail-biting pens (Ursinus, 2015), the authors investigated differences between the pigs from the barren or enriched-housing systems, but found no differences in the cortisol levels.

In the two social stressors investigated, we found breed-specific coping responses to social isolation stress on the farm and transport stress with LY pigs showing higher cortisol concentration in comparison with LYLD pigs. Although this corroborates previously reported results of breed differences in pigs (Li et al., 2008), it may be that our LY pigs generally responded more toward different stressors due to having a higher diurnal activity. The difference in the basal stress axis activity was found between sexes as well, with castrated males having higher activity than females.

Analysing time, we found different diurnal cortisol rhythm, as expected (see review; Chung et al., 2011). Morning concentrations were higher compared with the afternoon concentrations with no effect of day, so the response seemed to be equal each day under no apparent stressors. Furthermore, cortisol concentrations were higher before testing in the human test, while lower in the novelarena test. Knowing that social isolation is one of the most stressful situations for all social species including pigs, leading animals to suffer from separation anxiety (Forkman et al., 2007), we would expect an increase in cortisol concentrations after testing in both tests. Nevertheless, it seems that pigs perceived handling in their home pen, before being led to the isolation pen, as more aversive than testing itself. Studies addressing handling effects on pig behaviour (e.g. Oliveira et al., 2015) found that pigs can discriminate between handlers and if animals are not habituated to the handler, the main consequence is the increased fear, which probably is what happened in our study. Next to social isolation, transport is perceived as highly stressful for pigs. It is known that if the stimulus is maintained for some time, circulating levels of corticoid hormones return to baseline as a result of readjustments of adaptation mechanisms, allostasis (Korte et al., 2005). The same was seen in our study with pigs having the lowest concentrations before they were transported and the highest $60 \mathrm{~min}$ after the beginning of the transport. Afterwards, the concentration started to drop toward the basal concentration.

Although it is unclear to us why, in the case of both phenotypes and genotypes, autonomic nervous system was activated in a different matter while HPA-axis remained unchanged in most testing situations, controversial results were reported elsewhere, e.g., cattle: Kovács et al. (2015); and humans: Lupien (2009). In pigs, the animals that were handled unpleasantly showed more fearful behaviour with concomitant no depressed growth rates or cortisol responses of stress. In humans, the author argued that an emotion such as fear can create bodily reactions that are similar to those induced by a stressor (e.g. an increase in heart rate), but does not necessarily elicit a stress reaction. When considering challenges existing in commercial housing systems with tail-biting outbreaks, our previous results imply these challenges to lead to psychological dysfunction of some pigs (Zupan et al., 2012) and, according to Valros et al. (2013), to a blunted stress response, which is a sign of hypocortisolism. This leads us to think that biting tails and having tails bitten will most likely lead to some degree of damage in the regulation of both ANS and HPA-axis. Regardless of the explanation for the gained results in our study, it is clear that salivary cortisol concentrations were less informative than that of cardiovascular activity in pigs with behavioural problems that were subjected to a prolonged or a repeated stress in the form of tail biting.

\section{Conclusions}

Based on the investigation of stress physiology by characterizing cortisol responses to stressors in tail-biters, victims, and control pigs, we conclude that pigs consider the exposure to a novel arena, but not to a novel object, stressful. Also, genotype affects the psycho-physiological state of pigs from tail-biting pens since salivary cortisol responses differ between sexes and breeds.

\section{Acknowledgments}

This project was funded by the Nordic Joint Committee for Agricultural and Food Research and the Norwegian Research Council, project number 188520, awarded to Adroaldo José Zanella. For the preparation of the manuscript, funds were provided to Adroaldo José Zanella by the Conselho Nacional de Desenvolvimento Científico e Tecnológico (award 312985/2013-4). The Department of Preventive Veterinary Medicine and Animal Health, School of Veterinary Medicine and Animal Science, University of São Paulo, contributed with resources from CAPES. The European Economic Area grant provided Manja Zupan with a financial help for engagement in the project. A special "thank you" goes to Andrew Janczak, who was involved in data collection and discussion about the study. We are also grateful to Tore Framstad, Carlos Ventura, Hanna Lind Johansen, and Kristian Ellingsen for their 
assistance. Marjorie Coulon is acknowledged for providing the initial help with the ELISA. Special thanks also go to the Norwegian farmers who were willing to cooperate.

\section{References}

Chung, S.; Son, G. H. and Kim, K. 2011. Circadian rhythm of adrenal glucocorticoid: Its regulation and clinical implications. Biochimica et Biophysica Acta (BBA) - Molecular Basis of Disease 1812:581-591.

Forkman, B.; Boissy, A.; Meunier-Salaün, M. C.; Canali, E. and Jones, R. B. 2007. A critical review of fear tests used on cattle, pigs, sheep, poultry and horses. Physiology \& Behavior 92:340-374.

Fries, E.; Hesse, J.; Hellhammer, J. and Hellhammer, D. H. 2005. A new view on hypocortisolism. Psychoneuroendocrinology 30:1010-1016.

Heinonen, M.; Orro, T.; Kokkonen, T.; Munsterhjelm, C.; Peltoniemi, O. and Valros, A. 2010. Tail biting induces a strong acute phase response and tail-end inflammation in finishing pigs. The Veterinary Journal 184:303-307.

Herman, J. P.; Ostrander, M. M.; Mueller, N. K. and Figueiredo, H. 2005. Limbic system mechanisms of stress regulation: hypothalamo-pituitary-adrenocortical axis. Progress in NeuroPsychopharmacology \& Biological Psychiatry 29:1201-1213.

Korte, S. M.; Koolhaas, J. M.; Wingfield, J. C. and McEwen, B. S. 2005. The Darwinian concept of stress: benefits of allostasis and costs of allostatic load and the trade-offs in health and disease. Neuroscience \& Biobehavioral Reviews 29:3-38.

Kovács, L.; Kézér, F. L.; Jurkovich, V.; Kulcsár-Huszenicza, M. K. and Tözsér, J. 2015. Heart rate variability as an indicator of chronic stress caused by lameness in dairy cows. PLoS One 10: e 0134792. doi: 10.1371/journal.pone.0134792.

Li, L. A.; Xia, D.; Bao, E. D.; Wei, S.; Xiao, J. S.; Bao, J. W.; Chen, W. H.; Chen, J.; Hartung, J. and Zhao, R. Q. 2008. Erhualian and Pietrain pigs exhibit distinct behavioural, endocrine and biochemical responses during transport. Livestock Production Science 113:169-177.

Lupien, S. J. 2009. Brains under stress. Canadian Journal of Psychiatry 54:4-5.

Oliveira, D.; Paranhos da Costa, M. J. R.; Zupan, M.; Rehn, T. and Keeling, L. J. 2015. Early human handling in non-weaned piglets: Effects on behaviour and body weight. Applied Animal Behaviour Science 164:56-63.

Sautron, V.; Terenina, E.; Gress, L.; Lippi, Y.; Billon, J.; Larzul, C.; Liaubet, L.; Villa-Vialaneix, N.and Mormède, P. 2015. Time course of the response to ACTH in pig: biological and transcriptomic study. BMC Genomics 16:961.

Taylor, N. R.; Main, D. C. J.; Mendl, M. and Edwards, S. A. 2010. Tail biting: a new perspective. The Veterinary Journal 186:137-147.

Ursinus, W. W.; Van Reenen, C. G.; Reimert, I. and Bolhuis, J. E. 2014. Tail biting in pigs: Blood serotonin and fearfulness as pieces of the puzzle? PLoS One 9:9.

Valros, A.; Munsterhjelm, C.; Puolanne, E.; Ruusunen, M.; Heinonen, M.; Peltoniemi, O. A. T. and Pösö, A. R. 2013. Physiological indicators of stress and meat and carcass characteristics in tail bitten slaughter pigs. Acta Veterinaria Scandinavica 55:75.

Zupan, M.; Framstad, T. and Zanella, A. J. 2016. Behaviour, heart rate and heart rate variability in pigs exposed to novelty. Revista Brasileira de Zootecnia 45:121-129.

Zupan, M.; Janczak, A. M.; Framstad, T. and Zanella, A. J. 2012. The effect of biting tails and having tails bitten in pigs. Physiology \& Behaviour 106:638-644. 